\title{
Survival time and effect of selected predictor variables on survival in owned pet cats seropositive for feline immunodeficiency and leukemia virus attending a referral clinic in northern Italy
}

\author{
Eva Spada*, Roberta Perego, Elena Assunta Sgamma, Daniela Proverbio \\ Department of Veterinary Medicine (DIMEVET), University of Milan, Via G. Celoria 10, 20133 Milan, Italy
}

\section{A R T I C L E IN F O}

\section{Keywords:}

Feline

FIV

FeLV

Seropositivity

Survival time

Predictor variable

\begin{abstract}
A B S T R A C T
Feline immunodeficiency virus (FIV) and feline leukemia virus (FeLV) are among the most important feline in fectious diseases worldwide.

This retrospective study investigated survival times and effects of selected predictor factors on survival time in a population of owned pet cats in Northern Italy testing positive for the presence of FIV antibodies and FeLV antigen.

One hundred and three retrovirus-seropositive cats, 53 FIV-seropositive cats, 40 FeLV-seropositive cats, and 10 FIV + FeLV-seropositive cats were included in the study. A population of 103 retrovirus-seronegative age and sex-matched cats was selected. Survival time was calculated and compared between retrovirus-seronegative, FIV, FeLV and FIV + FeLV-seropositive cats using Kaplan-Meier survival analysis. Cox proportional-hazards regression analysis was used to study the effect of selected predictor factors (male gender, peripheral blood cytopenia as reduced red blood cells - RBC- count, leukopenia, neutropenia and lymphopenia, hypercreatininemia and reduced albumin to globulin ratio) on survival time in retrovirus-seropositive populations.

Median survival times for seronegative cats, FIV, FeLV and FIV + FeLV-seropositive cats were 3960, 2040, 714 and 77 days, respectively. Compared to retrovirus-seronegative cats median survival time was significantly lower $(P<0.000)$ in FeLV and FIV + FeLV-seropositive cats. Median survival time in FeLV and FIV + FeLV-seropositive cats was also significant lower $(P<0.000)$ when compared to FIV-seropositive cats. Hazard ratio of death in FeLV and FIV + FeLV-seropositive cats being respectively 3.4 and 7.4 times higher, in comparison to seronegative cats and 2.3 and 4.8 times higher in FeLV and FIV + FeLV-seropositive cats as compared to FIV-seropositive cats.

A Cox proportional-hazards regression analysis showed that FIV and FeLV-seropositive cats with reduced RBC counts at time of diagnosis of seropositivity had significantly shorter survival times when compared to FIV and FeLV-seropositive cats with normal RBC counts at diagnosis.

In summary, FIV-seropositive status did not significantly affect longevity of cats in this study, unlike FeLV and FIV + FeLV-seropositivity. Reduced RBC counts at time of FIV and FeLV diagnosis could impact negatively on the longevity of seropositive cats and therefore blood counts should always be evaluated at diagnosis and follow-up of retrovirus-seropositive cats.
\end{abstract}

\section{Introduction}

The two feline retroviruses, feline immunodeficiency virus (FIV) and feline leukemia virus (FeLV), are global and widespread, but differ in their potential to cause disease.

FIV, a retrovirus of the genus Lentivirus, can cause an acquired immune deficiency syndrome, which predisposes cats to other infections.
Clinical signs are most often a reflection of opportunistic infections, neoplasia and/or myelosuppression. However, in most naturally infected cats, FIV does not cause a severe clinical syndrome; with appropriate care, FIV-infected cats can live many years before succumbing to conditions unrelated to their FIV infection. Thus, overall survival time is not necessarily shorter than in uninfected cats, and quality of life is usually high over many years or lifelong (Addie et al., 2000; Hofmann-Lehmann et al., 1997; Levy et al., 2008).

\footnotetext{
* Corresponding author.

Email address: eva.spada@unimi.it (E. Spada)
} 
FeLV, a $\gamma$-retrovirus member of the Oncornavirus subfamily of retroviruses, is more pathogenic than FIV. Progressive FeLV infection can cause tumors, bone marrow suppression and immunosuppression, as well as neurological and other disorders, resulting in decreased life expectancy (Lutz et al., 2009). In contrast to FIV, persistent FeLV infection has a much greater effect on life expectancy of infected cats (Addie et al., 2000; Hofmann-Lehmann et al., 1997). However, with appropriate care, many FeLV-infected cats can also live for several years after diagnosis with a good quality of life (Helfer-Hungerbuehler et al., 2015; Hofmann-Lehmann et al., 1995; Levy et al., 2008).

Many studies have explored the risk factors for seropositivity and clinic-pathological features for retrovirus infected cats. Amongst these risk factors and features, male sex (Arjona et al., 2000; Burling et al., 2017; Chhetri et al., 2015; Garigliany et al., 2016; Gates et al., 2017; Gleich et al., 2009a,b; Levy et al., 2006; Liem et al., 2013; Murray et al., 2009; Peri et al., 1994; Ravi et al., 2010; Spada et al., 2012; Westman et al., 2016) and peripheral blood cytopenias such as anemia, leukopenia with neutropenia and lymphopenia are a common and well documented findings for both FIV and FeLV infections (Ackley et al., 1990; Arjona et al., 2000; Fujino et al., 2009; Gleich and Hartmann, 2009; Hofmann-Lehmann et al., 1997; Kohmoto et al., 1998; Shelton et al., 1995; Spada et al., 2012; Sparkes et al., 1993). In addition FIV-infected cats can develop an excessive immune response leading to hypergammaglobulinemia, reduced albumin to globulin ratio $(A / G)$ and hyperproteinemia (Gleich and Hartmann, 2009; Hofmann-Lehmann et al., 1997; Liem et al., 2013; Mirò et al., 2007) which reflects polyclonal B-cell stimulation (Ackley et al., 1990; Flynn et al., 1994; Gleich and Hartmann, 2009; Shelton et al., 1995; Sparkes et al., 1993). Finally renal involvement can occur in a proportion of naturally and experimentally FIV-infected cats (Baxter et al., 2012; Poli et al., 2012).

A limited number of studies have reported survival times for retrovirus infected cats, compared survival time to uninfected cats and studied the effect of predictor factors on survival times both for FeLV (Addie et al., 2000; Chhetri et al., 2015; Gleich et al., 2009b; Levy et al., 2008) and for FIV-infected cats (Addie et al., 2000; Chhetri et al., 2015; Gleich et al., 2009b; Levy et al., 2008; Liem et al., 2013; Ravi et al., 2010). In addition, results of studies on survival time may differ geographically as the clinical course of retrovirus infection is determined by a combination of viral and host factors. Some of these differences can be traced to properties of the virus itself, such as the subgroup that determines differences in the clinical picture. For example FeLV-B is primarily associated with tumors, FeLV-C is primarily associated with non-regenerative anemia (Dean et al., 1992). Different FIV subtypes exist, and subtype B is a less virulent strain of FIV than subtype A (Bachmann et al., 1997; Kohmoto et al., 1998). Therefore, there may be geographical/strain-related variations in clinical signs, diseases, and opportunistic infections associated with retrovirus infections.

The aims of this study were to estimate the survival times and to explore the effect of selected predictor factors on survival times in owned pet cats that were tested for FIV antibodies and FeLV antigen for a variety of medical reasons at a referral clinic in northern Italy over a 14 years period. Our hypothesis was that the type of retrovirus might alter the effect on survival time and that some risk factors for retrovirus seropositivity could also have a prognostic value for survival.

\section{Material and methods}

A retrospective cohort study was designed. Medical records of cats referred for different medical reasons to the Veterinary Clinic of the University of Milan were examined retrospectively to identify cats that had been tested for FIV antibodies and FeLV antigen, between January 2002 and the first semester of 2016 (30 June 2016). Presence of antibody to FIV target antigens p24 and gp 40 and of FeLV p27 antigen were simultaneous checked on plasma, serum, or whole blood samples using a commercial rapid enzyme-linked immunosorbent assay (ELISA) kit (SNAP ${ }^{\circledR}$ Combo Plus FeLV Ag/FIv Ab, IDEXX Laboratories, Europe). This ELISA test kit showed excellent performance in diagnosis of these retroviruses with a sensitivity and specificity for FeLV diagnosis of $100 \%$ and a sensitivity and specificity for FIV diagnosis of $97.9 \%$ and $99.0 \%$, respectively (Levy et al., 2017).

FIV and FeLV-seropositive cats formed the study population. A comparison group of retrovirus-seronegative cats with similar sex and age characteristics was created. For each retrovirus-seropositive cat, one retrovirus-seronegative was enrolled in the study. This retrovirus-seronegative cat was selected as the first cat of the same sex and of similar age tested in the same day or month or year (within 1 year). If age and sex matching were not simultaneously available matching was performed based only on one of the two parameters, depending on which was available.

For each retrovirus-seronegative and seropositive cat, data retrieved from medical records were: data of the first FIV/FeLV test, age at test, sex, reason for FIV-FeLV test (pre-FeLV vaccination, pre-neuter check, pre-blood donor check, unhealthy cat), hematological and biochemical data, details of death/euthanasia, clinical condition in alive cats.

Laboratory data collected at time of test at admission were: complete blood count (CBC) using ADVIA 120 analyser (Bayer) until end of may 2010 and Cell-Dyn 3500 haematology analyser (Abbott Diagnostic Laboratories) from July 2010 to the end of the study comprising red blood cells (RBCs), hemoglobin (Hb), hematocrit (Hct), mean cell volume (MCV), mean cell $\mathrm{Hb}(\mathrm{MCH})$, mean cell $\mathrm{Hb}$ concentration (MCHC), and white blood cells (WBCs). Differential leukocyte count was made by blood smear evaluation (microscopic magnification $100 \mathrm{x}$ immersion objective) to make a quantitative evaluation of the percentage in 10 fields.

Biochemical data collected comprised: serum creatinine (by cinetic modified Jaffè method) and total serum protein (by biuret colorimetric method) evaluated using Cobas Mira Classics analyser (Roche). Alfa, beta and gammaglobulins, albumin to globulin $(\mathrm{A} / \mathrm{G})$ ratio were evaluated by agarose gel serum electrophoresis (Hydrasys-Sebia). Laboratory reference ranges used were taken from the literature (Giordano and Paltrinieri, 2010; Moritz et al., 2004; Silverstein and Hopper, 2015).

\subsection{Statistical analysis}

All the collected data were captured in Microsoft Excel 2007 and analyzed using statistical software (MedCalc statistical software version 16.4.3 and SPSS statistics software, version 18.0).

Descriptive statistics were presented to define the population and survival predictor variables for cats at entry into the study.

Survival times were calculated from the date of diagnosis of retroviral seropositivity to the date of death or euthanasia. If these data were not available, the owner was contacted by telephone to request follow-up information. If the cat had died and the exact date of death was unknown, the month of death was recorded and it was assumed that the cat died on the 15th of that month for the purposes of the survival analysis. Cats were censored in the survival analysis if they were still alive at the end of the follow-up period (June 30, 2016) or if they were lost to follow-up. Cats were categorized as lost to follow up if they had not attended the clinic for 6 months and their owners were not contactable by telephone (after three calls made on three different days at three different times of day).

Differences between the median age of the groups were tested for significance using the non-parametric Mann-Whitney $U$ test for unpaired samples.

Kaplan-Meier survival analyses were performed to estimate survival of retrovirus-seronegative and seropositive cats groups (ie FIV, FeLV or FIV + FeLV-seropositive cats). Pairwise comparison of survival curves was performed using the Mantel-Cox logrank test. The hazard ratios 
with $95 \% \mathrm{CI}$ (ie how rapidly death occurred in the groups) were also calculated (Klein and Moeschberger, 2003).

Patients lost to follow-up or still alive at the time of follow-up were censored by the date on which they were last seen by the diagnosing veterinarian or the last date known to be alive.

Effects of selected predictor variables on survival were analyzed using a Cox proportional hazard model. The following variables analyzed as dichotomous (code 1 if present and 0 if absent), were entered into the proportional-hazards regression Cox model to determine if they were independently associated with survival of retrovirus-seropositive cats: male gender, reduced RBC count, reduced WBC count, neutropenia, lymphopenia, increased creatinine and serum A/G ratio and are presented with the $95.0 \%$ confidence interval. These variables were chosen as relevant risk factors for seropositive status and well documented pathological alterations identified in retrovirus infected cats (Ackley et al., 1990; Arjona et al., 2000; Baxter et al., 2012; Burling et al., 2017; Chhetri et al., 2015; Flynn et al., 1994; Garigliany et al., 2016; Gates et al., 2017; Gleich and Hartmann, 2009; Gleich et al., 2009a,b; Hofmann-Lehmann et al., 1997; Levy et al., 2006; Liem et al., 2013; Mirò et al., 2007; Murray et al., 2009; Peri et al., 1994; Poli et al., 2012; Ravi et al., 2010; Shelton et al., 1995; Spada et al., 2012; Sparkes et al., 1993; Westman et al., 2016). Univariable Cox regression analysis was performed initially. Variables with a $P$ value $<0.2$ in the univariate analysis subsequently were entered into a forward selection, stepwise multivariable Cox regression analysis with significance set at $P<0.05$.

\section{Results}

The total number of cats tested during the years 2002 until first semester 2016 (30 June 2016) was 816 owned pet cats out of 1600 owned pet cats evaluated in the same period.

Out of 816 cats tested, 117 owned cats tested positive for retrovirus infection (14.3\%, 95.0\% CI 11.8-17.1). Of these 60 cats were FIV-positive giving an estimated prevalence of FIV seropositivity of $7.4 \%$ (95.0\% CI 5.6-9.4), 46 cats were FeLV-seropositive giving an estimated prevalence of $5.6 \%$ (95.0\% CI 4.1-7.5) and 11 cats $(1.4 \%, 95.0 \%$ CI $0.6-2.4)$ were seropositive for both FIV and FeLV.

For 14 retrovirus-seropositive cats (7 FIV-seropositive cats, 6 FeLV-seropositive cats, and 1 FIV + FeLV-seropositive cat) medical records were missing. These cats were excluded from the analysis, therefore 103 retrovirus-seropositive cats were analyzed in this study, of which 53 were FIV-seropositive cats (51.0\%), 40 FeLV-seropositive cats (39.0\%) and 10 FIV + FeLV-seropositive cats (10.0\%).

Of these 103 retrovirus-seropositive cats, 59 were male and 44 were female. In particular 34 males and 19 females were FIV-seropositive cats, 19 males and 21 females were FeLV-seropositive cats and 6 males and 4 females were FIV + FeLV-seropositive cats. Age was recorded for 93 cats, with a median value of 4.0 years $(95.0 \%$ CI $3.0-5.0$, range 3 months-16 years).

Age at diagnosis of retrovirus-positive status was recorded for $47 /$ 53 FIV-seropositive, 39/40 FeLV-seropositive cats and for 7/10 FIV + FeLV-seropositive cats, with a median value of 5.0 years $(95.0 \%$ CI 3.6-7.0, range 6 months-16 years), 2.0 years (95.0\% CI 1.0-4.0, range 3 months- 13 years), and 7.0 years (95.0\% CI 5.0-11.0, range 5.0-11.0 years) respectively. At Mann-Whitney $U$ test median age was significantly higher in FIV-seropositive than in FeLV-seropositive cats $(P=0.0002)$, but not higher than FIV + FeLV-seropositive cats $(P=0.1797)$. Median age in FELV-seropositive cats resulted significantly lower than in FIV + FeLV-seropositive cats $(P=0.0026)$.

No cat was anesthetized or sedated to collect blood samples for the diagnosis of FIV or FeLV seropositivity.
Descriptive statistics relative to age group, cause for retrovirus test, cause of death, clinical status in alive cats and potential risk factors for survival in retrovirus-positive cats divided into groups of: FIV-seropositive cats, FeLV-seropositive cats and FIV + FeLV-seropositive cats are presented in Table 1.

A population of 103 sex and/or age-matched retrovirus seronegative cats was selected, of which 58 were male and 43 female. Median age was 4.0 years ( $95.0 \%$ CI 3.0-5.9, range 4 months- 16 years). There was no significant difference between median age of retrovirus-seronegative cats and retrovirus-seropositive cats $(P=0.9456)$ at Mann-Whitney $U$ test. No statistical differences were found between the median age of retrovirus-seronegative FIV-seropositive cats and FIV + FeLV-seropositive cats $(P=0.101$ and $P=0.065$, respectively), but there was a significant difference between retrovirus-seronegative and FeLV-seropositive cats $(P=0.008)$.

Descriptive statistics relative to age group, cause for retrovirus test, cause of death, clinical status in alive/surviving cats and potential risk factors for survival in retrovirus-seronegative cats are presented in Table 1.

Summary statistic relating to hematological and biochemical parameters measured at admission when retrovirus test was performed are reported in Table 2.

The mean and median survival times of retrovirus-seronegative cats, FIV-seropositive, FeLV-seropositive and FIV + FeLV-seropositive cats are reported in Table 3 and in Fig. 1.

The type of retroviral infection had a significant influence on survival time. In fact when compared to retrovirus-seronegative cats survival time was significantly lower in FeLV and FIV + FeLV-seropositive cats (Chi-Square $=16.4$ and 27.3 with $P=0.000$ and $P=0.000$, respectively), bur not significantly different in FIV-seropositive cats (Chi-Square $=1.2, P=0.260$ ). The survival time of FIV-seropositive cats was significantly greater than in FeLV and FIV + FeLV-seropositive cats (Chi-Square $=7.0$ and 16.9 with $P=0.008$ and $P=0.000$, respectively). Survival time in FeLV-seropositive cats was not significantly different from FIV + FeLV-seropositive cats (Chi-Square $=2.9$, $P=0.083$ ).

The hazard ratio for death in FeLV and FIV + FeLV seropositive cats was 3.4 (95.0\% CI 1.6-6.9) and 7.4 (95.0\% CI 1.5-36.7) respectively when compared with retrovirus-seronegative cats and $2.3(95.0 \% \mathrm{CI}$ $1.1-4.9)$ and 4.8 (95.0\% CI 1.2-18.5) respectively when compared to FIV-seropositive cats.

Results of univariable Cox regression analysis of potential factors associated with survival at admission for FIV-seropositive, FeLV-seropositive and FIV + FeLV-seropositive cats are reported in Tables 4-6.

The multivariable Cox proportional-hazards regression model for variables that could predict the survival time for FIV-seropositive cats included factors for reduced RBCs count, neutropenia and reduced A/G ratio (Table 4). Neutropenia and reduced $\mathrm{A} / \mathrm{G}$ ratio were found in multivariate analysis not to be significantly associated with survival time in FIV-seropositive cats. In the final model only reduced RBCs count was independently associated with survival in multivariable analysis (Table 7, Fig. 2). Hazard ratio for reduced RBC counts in FIV-seropositive cats was 11.0 meaning that a cat with reduced a RBC count at diagnosis of FIV seropositivity had a death ratio 11 times higher than a cat with normal RBC count.

In FeLV-seropositive cats Cox proportional-hazards univariable regression found that the variable reduced RBC count predicted the survival time (Table 7, Fig. 3). Hazard ratio for reduced RBC counts in FeLV-seropositive cats was 3.5 meaning that a cat with a reduced RBC count at diagnosis of FeLV seropositivity had a death ratio 3.5 higher than a cat with normal RBCs count. Effects of male gender, reduced lymphocyte, neutrophil and total WBC count, increased creatinine and 
Table 1

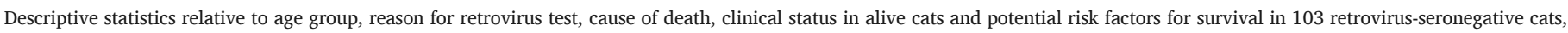
53 FIV-seropositive cats, 40 FeLV-seropositive cats and 10 FIV + FeLV-seropositive cats.

\begin{tabular}{|c|c|c|c|c|c|}
\hline \multirow[t]{2}{*}{ Parameter } & & \multicolumn{3}{|l|}{ Group } & \multirow[b]{2}{*}{$\begin{array}{l}\text { FIV + FeLV- } \\
\text { seropositive }\end{array}$} \\
\hline & & $\begin{array}{l}\text { Retrovirus-seronegative } \\
\text { cats }\end{array}$ & $\begin{array}{l}\text { FIV- } \\
\text { seropositive }\end{array}$ & $\begin{array}{l}\text { FeLV- } \\
\text { seropositive }\end{array}$ & \\
\hline \multirow[t]{6}{*}{ Age group (years) } & $<1$ & $16(15.5 \%)$ & $3(5.7 \%)$ & $7(17.5 \%)$ & $0(0 \%)$ \\
\hline & $1-5$ & $45(43.8 \%)$ & $23(43.4 \%)$ & $24(60.0 \%)$ & $2(20.0 \%)$ \\
\hline & $>5-10$ & $26(25.2 \%)$ & $19(35.9 \%)$ & $6(15.0 \%)$ & $5(50.0 \%)$ \\
\hline & $>10-15$ & $14(13.6 \%)$ & $6(11.4 \%)$ & $3(7.5 \%)$ & $3(30.0 \%)$ \\
\hline & $>15$ & $2(1.9 \%)$ & $1(1,8 \%)$ & $0(0.0 \%)$ & $0(0.0 \%)$ \\
\hline & NR & $0(0.0 \%)$ & $1(1,8 \%)$ & $0(0.0 \%)$ & $0(0.0 \%)$ \\
\hline \multirow[t]{4}{*}{ Reason for retrovirus test } & Pre-FeLV vaccination & $7 / 103(6.8 \%)$ & $4 / 53(7.6 \%)$ & $5 / 40(12.5 \%)$ & $0 / 10(0.0 \%)$ \\
\hline & Pre-neuter check & $24 / 103(23.3 \%)$ & $\begin{array}{l}11 / 53 \\
(20.7 \%)\end{array}$ & $10 / 40(25.0 \%)$ & $1 / 10(10.0 \%)$ \\
\hline & Pre-blood donor check & 10/103 (9.7\%) & $8 / 53(15.1 \%)$ & $0 / 40(0.0 \%)$ & $0 / 10(0.0 \%)$ \\
\hline & $\begin{array}{l}\text { Unhealthy (various } \\
\text { illness) }\end{array}$ & $62 / 103(60.2 \%)$ & $\begin{array}{l}30 / 53 \\
(56.6 \%)\end{array}$ & $25 / 40(62.5 \%)$ & $9 / 10(90.0 \%)$ \\
\hline \multirow[t]{4}{*}{ Cause of death } & Anemia & $2 / 19(10.5 \%)$ & $6 / 17(35.3 \%)$ & $5 / 20(25.0 \%)$ & 4/7 (57.1\%) \\
\hline & Neoplasia & $5 / 19(26.4 \%)$ & $2 / 17(11.8 \%)$ & $11 / 20(55.0 \%)$ & $2 / 7(28.6 \%)$ \\
\hline & Other & $10 / 19(52.6 \%)$ & $3 / 17(17.6 \%)$ & $2 / 20(10.0 \%)$ & $0 / 7(0.0 \%)$ \\
\hline & NR & $2 / 19(10.5 \%)$ & $6 / 17(35.3 \%)$ & $2 / 20(10.0 \%)$ & $1 / 7(14.3 \%)$ \\
\hline \multirow[t]{3}{*}{ Clinical status of alive cats } & Healthy & $22 / 84(26.2 \%)$ & $\begin{array}{l}12 / 17 \\
(70.6 \%)\end{array}$ & $4 / 10(40.0 \%)$ & None alive \\
\hline & Unhealthy & $61 / 84(72.6 \%)$ & $4 / 17(23.5 \%)$ & $2 / 10(20.0 \%)$ & None alive \\
\hline & NR & $1 / 84(1.2 \%)$ & $1 / 17(5.9 \%)$ & $4 / 10(40.0 \%)$ & None alive \\
\hline \multirow{7}{*}{$\begin{array}{l}\text { Presence of potential risk factor for } \\
\text { survival }\end{array}$} & Reduced RBC count & 13/103 (12.6\%) & $11 / 53$ & $14 / 40(35.0 \%)$ & $6 / 10(60.0 \%)$ \\
\hline & Leucopenia & $22 / 103(21.3 \%)$ & $18 / 53$ & $14 / 40(35.0 \%)$ & $3 / 10(30.0 \%)$ \\
\hline & Neutropenia & $17 / 103(16.5 \%)$ & $11 / 53$ & $9 / 40(22.5 \%)$ & $1 / 10(10.0 \%)$ \\
\hline & Iymphonenis & $15 / 103(14.5 \%)$ & $6 / 53(113 \%)$ & $4 / 40(100 \%)$ & $1 / 10(10.0 \%)$ \\
\hline & Hypercreatinemia & $43 / 103(41.7 \%)$ & $21 / 53$ & $9 / 40(22.5 \%)$ & $4 / 10(40.0 \%)$ \\
\hline & & & $(39.6 \%)$ & & \\
\hline & Reduced A/G ratio & 28/103 (27.1\%) & $\begin{array}{l}20 / 53 \\
(37.7 \%)\end{array}$ & $11 / 40(27.5 \%)$ & $4 / 10(40.0 \%)$ \\
\hline
\end{tabular}

NR: not recorded in the medical records.

reduced $\mathrm{A} / \mathrm{G}$ ratio on survival time were not significant and were not included in the model .

\section{Discussion}

This study investigated survival time in retrovirus-seropositive owned pet cats of northern Italy and found survival data were in agreement with previous studies (Addie et al., 2000; Gleich et al., 2009a,b; Levy et al., 2008; Liem et al., 2013; Ravi et al., 2010). Notably, FIV-infected cats have an apparently normal life expectancy, not statistically different to retrovirus-seronegative cats. This is possibly related to the long incubation period for this virus as cats may remain relatively disease-free for 8 years or more (Kohmoto et al., 1998).

Median survival time of FIV-seropositive cats in our study (2040 days, 5.6 years) was greater than the 3.8 years (1416 days) reported for FIV-infected cats in Canada (Ravi et al., 2010), 4.9 years of FIV-infected cats in USA (Levy et al., 2008) and 2.0 years (748.8 days) of FIV-positive owned cats in Germany (Gleich et al., 2009a). This difference in survival time from previous studies should not be due to different ages at diagnosis, as the median age of FIV-seropositive cats in the present study was comparable to previous reports for FIV-infected cats (Gleich et al., 2009a; Levy et al., 2008; Ravi et al., 2010). This different survival time may be due to the subtype of virus affecting the feline population. FIV subtype B is prevalent in Italy (Pistello et al., 1997) and this subtype is less pathogenic in vitro and in vivo when compared with subtype A virus. Subtype B has been hypothesized to have a more advanced host adaptation and may, therefore, be less pathogenic than subtype A (Bachmann et al., 1997).

Differences in survival time could also be due to different management of FIV-seropositive cats of our population than of cats in other studies. In particular, housing conditions impact on disease progression and survival times of FIV-positive cats, with cats in large multi-cat household having shorter survival time than cats living in $<2$ cats group (Beczkowski et al., 2015). Although housing conditions were not recorded for cats in our study it is improbable that FIV-seropositive cats in our study were living in crowded multi-cat households, as they were owned pet cats from an urban area of Northern Italy.

In addition, our population of FIV infected cats had a significantly longer median survival time than FeLV infected cats, even if the median age at diagnosis of FIV-seropositive cats was statistically higher than median age of diagnosis for FeLV-seropositive cats. FeLV infection progresses more rapidly than FIV infection and is more pathogenic, so most cats that develop progressive infections ultimately die of FeLV-related disease (Sykes and Hartmann, 2014).

Survival time in FeLV-seropositive cats and FIV + FeLV-seropositive cats in our study was significantly shorter than that of retrovirus-seronegative cats. This finding is in agreement with previous studies that demonstrated a strong impact of FeLV on morbidity and mortality (Addie et al., 2000; Gleich et al., 2009a,b), typically as a consequence of FeLV-associated fatal diseases such as non-regenerative anemia, peripheral blood cytopenias and neoplasia (Helfer-Hungerbuehler et al., 2015; Shelton et al., 1990; Vail et al., 1998). In fact, similar to a previous study (Helfer-Hungerbuehler et al., 2015) a large proportion of deaths in FeLV-seropositive cats in our study seem to be related to FeLV as causes of death were mainly lymphoma and less frequently, anemia.

As previously reported in our study the median survival time after diagnosis of FeLV infection was less than 3 years (Addie et al., 2000; Helfer-Hungerbuehler et al., 2015; Levy et al., 2008). In the current study, the mean life expectancy of FeLV-seropositive cats was similar to 
Table 2

Summary statistics relating to selected hematological and biochemical parameters evaluated in 103 retrovirus-seronegative cats, 53 FIV-seropositive cats, 40 FeLV- seropositive cats and 10 FIV + FeLV-seropositive cats.

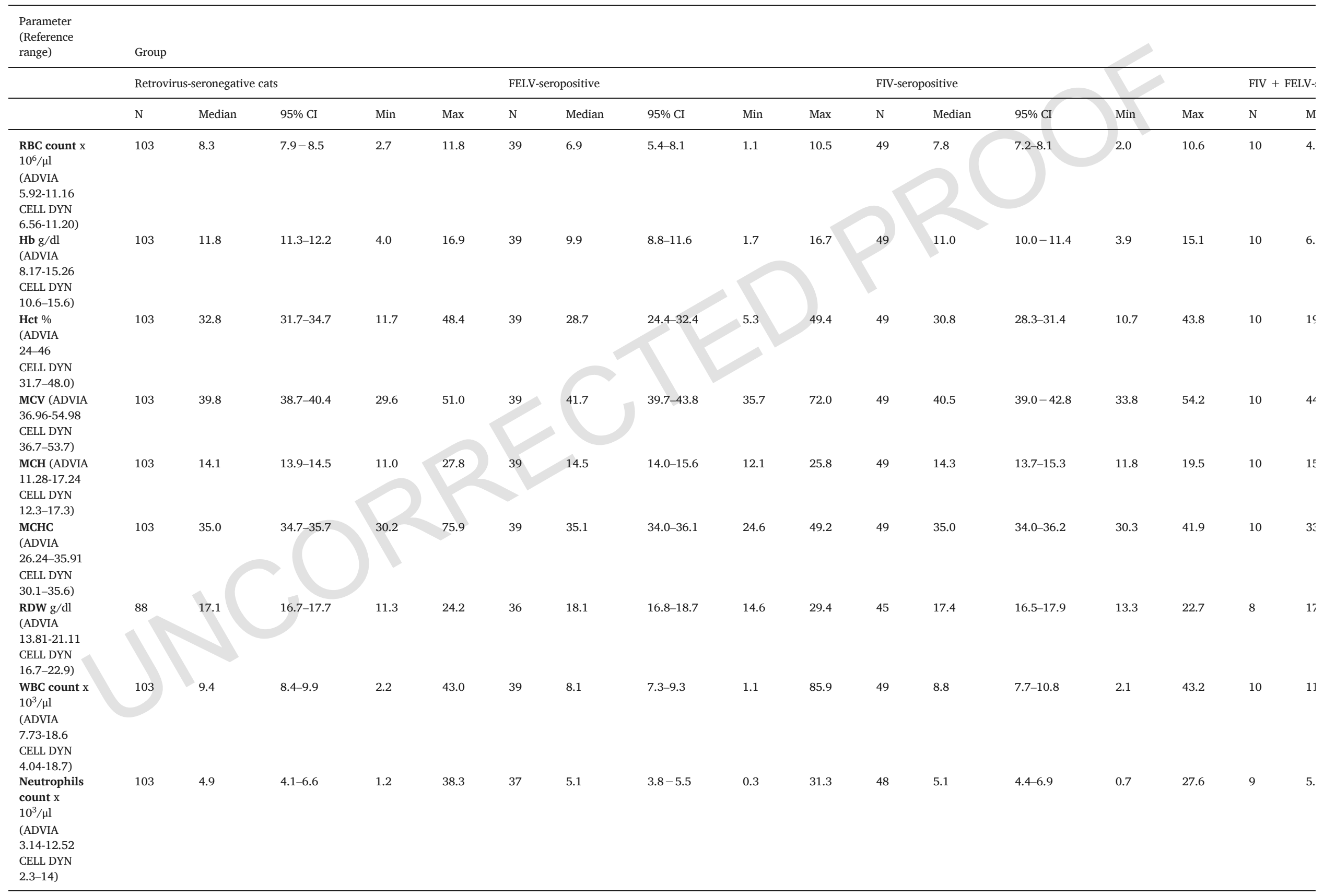


Table 2 (Continued)

\begin{tabular}{|c|c|c|c|c|c|c|c|c|c|c|c|c|c|c|c|c|c|}
\hline \multirow[t]{3}{*}{$\begin{array}{l}\text { Parameter } \\
\text { (Reference } \\
\text { range) }\end{array}$} & \multicolumn{17}{|c|}{ Group } \\
\hline & \multicolumn{5}{|c|}{ Retrovirus-seronegative cats } & \multicolumn{5}{|c|}{ FELV-seropositive } & \multicolumn{2}{|c|}{ FIV-seropositive } & \multirow[b]{2}{*}{$95 \%$ CI } & \multirow[b]{2}{*}{ Min } & \multirow[b]{2}{*}{ Max } & \multicolumn{2}{|c|}{ FIV + FELV- } \\
\hline & $\mathrm{N}$ & Median & $95 \% \mathrm{CI}$ & Min & Max & $\mathrm{N}$ & Median & $95 \% \mathrm{CI}$ & Min & Max & $\mathrm{N}$ & Median & & & & $\mathrm{N}$ & $\mathrm{M}$ \\
\hline $\begin{array}{l}\text { Lymphocyte } \\
\text { count }(\mathrm{x} \\
\left.10^{3} / \mu \mathrm{l}\right) \\
\text { (ADVIA } \\
1.31-7.46 \\
\text { CELL DYN } \\
0.8-6.1)\end{array}$ & 103 & 2.7 & $2.3-3.1$ & 0.3 & 12.8 & 36 & 2.7 & $2.2-3.4$ & 0.4 & 55.0 & 48 & 2.5 & $2.2-3.3$ & 0.4 & 9.5 & 9 & 4. \\
\hline $\begin{array}{l}\text { Creatinine } \\
\mathrm{mg} / \mathrm{dl} \\
(<1.5 \mathrm{mg} / \mathrm{dl})\end{array}$ & 89 & 1.5 & $1.5-1.6$ & 0.7 & 8.1 & 25 & 1.2 & $1.1-1.4$ & 0.5 & 2.4 & 39 & 1.6 & $1.3-1.8$ & 0.9 & 11.2 & 9 & 1. \\
\hline $\begin{array}{l}\text { Total protein } \\
\text { g/dl }(6-8)\end{array}$ & 98 & 7.1 & $6.8-7.3$ & 5.0 & 9.4 & 29 & 7.0 & $6.6-7.6$ & 5.2 & 8.9 & 43 & 7.1 & $6.8-7.4$ & 4.1 & 10.1 & 9 & 7. \\
\hline $\begin{array}{l}\text { Albumin } \mathrm{g} / \mathrm{dl} \\
(3.0-4.6)\end{array}$ & 92 & 3.4 & $3.3-3.5$ & 1.6 & 5.5 & 23 & 3.3 & $2.9-3.6$ & 2.5 & 4.9 & 39 & 3.3 & $3.2-3.6$ & 1.8 & 4.3 & 7 & 3. \\
\hline $\begin{array}{l}\text { Alfa } \\
\text { 1-globulins } \\
\text { g/dl } \\
(0.1-0.5)\end{array}$ & 91 & 0.2 & $0.2-0.2$ & 0.1 & 1.0 & 22 & 0.2 & $0.2-0.3$ & 0.1 & 1.4 & 33 & 0.2 & $0.2-0.3$ & 0.1 & 1.2 & 7 & 0. \\
\hline $\begin{array}{l}\text { Alfa } \\
\text { 2-globulins } \\
\text { g/dl } \\
(0.3-1.2)\end{array}$ & 90 & 1.1 & $1.0-1.1$ & 0.3 & 1.9 & 21 & 1.0 & $0.7-1.2$ & 0.3 & 2.7 & 32 & 0.9 & $0.7-1.1$ & 0.4 & 1.9 & 6 & 0. \\
\hline $\begin{array}{l}\text { Beta } \\
\text { 1-globulins } \\
\text { g/dl } \\
(0.1-0.7)\end{array}$ & 91 & 0.6 & $0.6-0.7$ & 0.1 & 1.6 & 21 & 0.5 & $0.4-0.6$ & 0.2 & 1.5 & 29 & 0.7 & $0.6-0.7$ & 0.2 & 1.0 & 7 & 0. \\
\hline $\begin{array}{l}\text { Beta } \\
\text { 2-globulins } \\
\text { g/dl } \\
(0.2-0.8)\end{array}$ & 77 & 0.4 & $0.3-0.5$ & 0.1 & 1.3 & 21 & 0.4 & $0.3-0.6$ & 0.2 & 0.9 & 30 & 0.5 & $0.4-0.6$ & 0.2 & 1.1 & 6 & 0. \\
\hline $\begin{array}{l}\text { Gamma- } \\
\text { globulins (g/ } \\
\text { dl) }(0.3-0.8)\end{array}$ & 91 & 1.2 & $1.0-1.3$ & 0.4 & 3.6 & 22 & 1.3 & $1.0-1.6$ & 0.6 & 2.2 & 34 & 1.6 & $1.3-2.0$ & 0.5 & 4.7 & 7 & 1. \\
\hline $\begin{array}{l}\text { A/G ratio }(\mathrm{g} / \\
\mathrm{dl}) \\
(0.8-1.6)\end{array}$ & 91 & 1.0 & $0.9-1.0$ & 0.4 & 1.7 & 23 & 0.9 & $0.8-1.1$ & 0.4 & 1.8 & 38 & 0.9 & $0.8-0.9$ & 0.3 & 1.5 & 7 & 0. \\
\hline
\end{tabular}

N: number; A/G: albumin to globulin; RBC: red blood cell; WBC: white blood cell; CI: confidence interval; Min: minimum; Max: maximum. 
Table 3

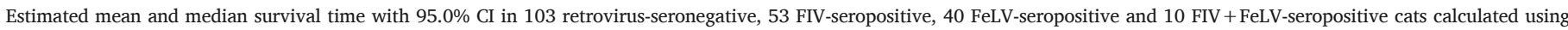
Kaplan Meier analysis.

\begin{tabular}{|c|c|c|c|c|c|}
\hline & \multicolumn{5}{|l|}{ Group } \\
\hline & $\begin{array}{l}\text { Retrovirus seronegative } \\
\text { cats }\end{array}$ & $\begin{array}{l}\text { FIV seropositive } \\
\text { cats }\end{array}$ & $\begin{array}{l}\text { FeLV seropositive } \\
\text { cats }\end{array}$ & $\begin{array}{l}\text { FIV + FeLV seropositive } \\
\text { cats }\end{array}$ & Overall \\
\hline n. of events (death) & $19 / 103$ & $17 / 53$ & $20 / 40$ & $7 / 10$ & $63 / 206$ \\
\hline n. of censored & $84 / 103$ & $36 / 53$ & $20 / 40$ & $3 / 10$ & $143 / 206$ \\
\hline n. of alive cats & $39 / 103$ & $17 / 53$ & $10 / 40$ & $0 / 10$ & $66 / 206$ \\
\hline n. of cats lost to follow up & $45 / 103$ & $19 / 53$ & $10 / 40$ & $3 / 10$ & $77 / 206$ \\
\hline Total sample size & 103 & 53 & 40 & 10 & 206 \\
\hline $\begin{array}{l}\text { Estimated mean survival time } \\
\text { (days) }\end{array}$ & 2822 & 2200 & 1113 & 396 & 2079 \\
\hline Standard error & 237 & 303 & 239 & 258 & 164 \\
\hline $95.0 \% \mathrm{CI}$ for the mean & $2357-3287$ & $1605-2795$ & $644-1582$ & $0-902$ & $1756-2402$ \\
\hline $\begin{array}{l}\text { Estimated median survival time } \\
\text { (days) }\end{array}$ & 3960 & 2040 & 714 & 77 & 2040 \\
\hline Standard error & 0.0 & 681 & 341 & 91 & 424 \\
\hline $95.0 \% \mathrm{CI}$ for the median & $0.0-0.0$ & $704-3375$ & $45-1382$ & $0-255$ & $1207-2872$ \\
\hline Range (min-max) & $0-3960$ & $0-4029$ & $0-3117$ & $0-1787$ & $0-4029$ \\
\hline
\end{tabular}

n. = number, $\mathrm{CI}=$ confidence interval, max: maximum, min: minimum.

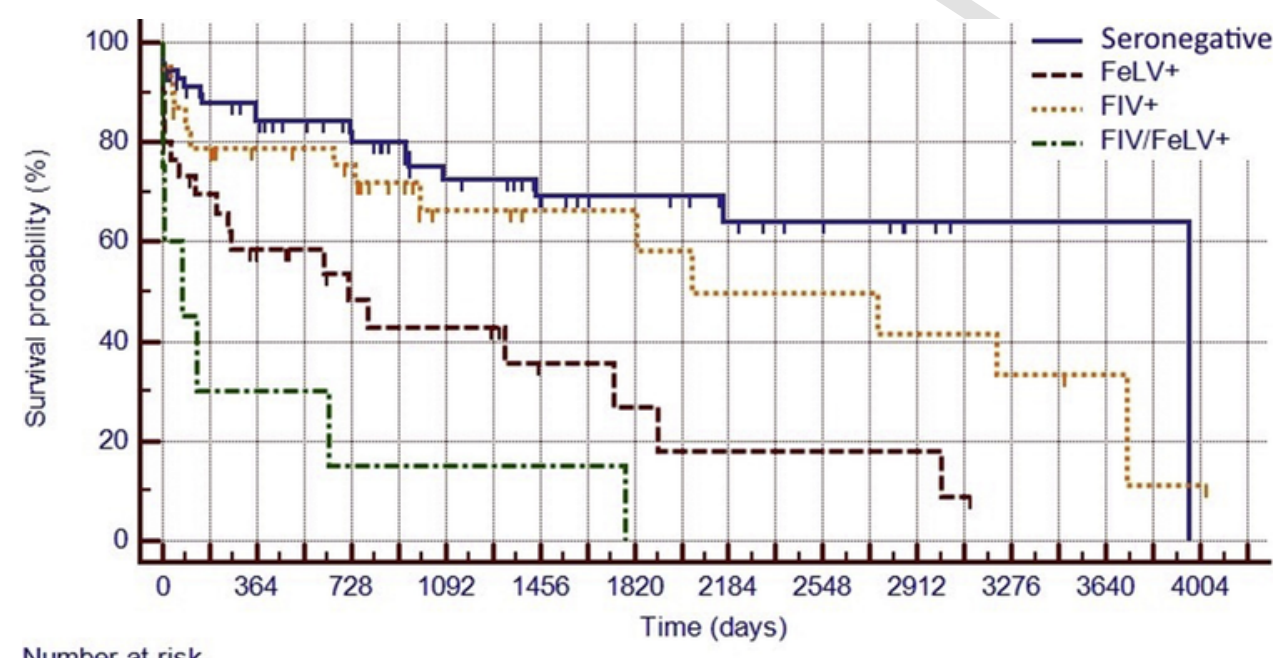

Number at risk

Group: Seronegative

$\begin{array}{llllllllllllllllllllllll}72 & 52 & 48 & 43 & 39 & 33 & 28 & 27 & 22 & 17 & 16 & 15 & 12 & 8 & 7 & 6 & 3 & 1 & 1 & 1 & 1 & 1 & 0 & 0\end{array}$

Group: FeLV+

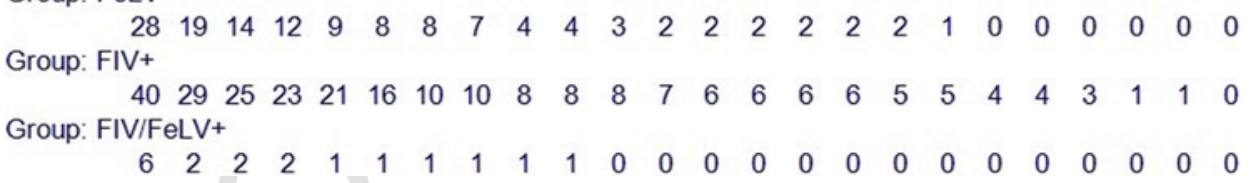

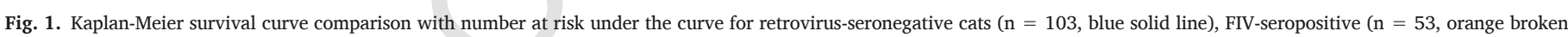

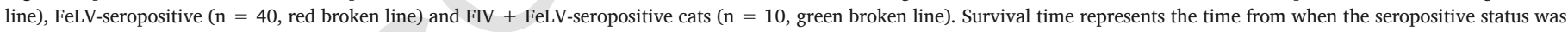

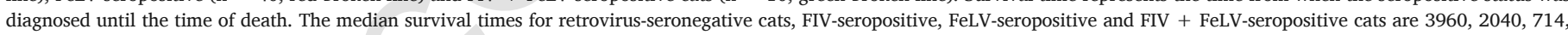

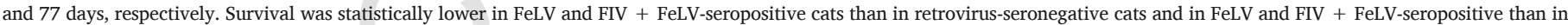

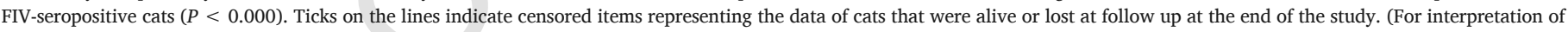
the references to colour in this figure legend, the reader is referred to the web version of this article.)

the 2.4 years reported in a large study by Levy et al. (2008). Despite this some FeLV-seropositive cats in our study lived up to 8.5 years (3117 days). This data highlights that FeLV infection should never be assumed to be a death sentence for the infected cat.

Co-infection with FeLV in FIV infected cats potentiates the primary and immune deficiency phases of FIV (Hofmann-Lehmann et al., 1995). Cats co-infected with FeLV and FIV have a poor prognosis as the retroviruses infect all lineages of hematopoietic and lymphoid cells with partially overlapping tropisms (Dean et al., 1992). This was confirmed by the reduced survival time of co-infected cats in our study and a death hazard ratio of 7.9 and 4.8 when compared to retrovirus-seronegative cats and FIV-seropositive cats, respectively. Pedersen et al.
(1990) also showed that infection with both FIV and FeLV leads to a more severe disease progression than FIV infection alone and that a preexisting FeLV infection enhanced the expression and spread of FIV in the body and increased the severity of both the resulting transient primary and chronic secondary stages of FIV infection (Pedersen et al., 1990).

Study of the potential predictor factors of survival in retrovirus-seropositive cats revealed that death occurred more rapidly in cats with reduced RBC counts at diagnosis in both FeLV and FIV infection. In FeLV-infected cats non-regenerative anemia is frequently the result of infection with subtype FeLV-C which results in pure red cell aplasia or aplastic anemia, myelodysplasia or myelofibrosis or immune-mediated 
Table 4

Results of univariable Cox regression analysis of potential factors at admission associated with survival at the $20 \%$ level $(P<0.2)$ of 53 FIV-seropositive cats.

\begin{tabular}{lllll}
\hline Variable & $95.0 \%$ CI for Hazard Ratio & & \\
\hline & $\begin{array}{l}\text { Hazard } \\
\text { Ratio }\end{array}$ & Lower & Upper & $\begin{array}{l}P \\
\text { value }\end{array}$ \\
& 1.199 & 0.431 & 3.334 & 0.729 \\
Male sex & 3.237 & 1.150 & 9.111 & $\mathbf{0 . 0 2 6}$ \\
Reduced RBCs & & & & \\
count & 0.493 & 0.159 & 1.159 & 0.220 \\
Leucopenia & 0.121 & 0.016 & 0.935 & $\mathbf{0 . 0 4 3}$ \\
Neutropenia & 1.212 & 0.339 & 4.335 & 0.768 \\
Lymphopenia & 0.984 & 0.315 & 3.076 & 0.978 \\
Hypercreatininemia & 3.602 & 0.946 & 13.713 & $\mathbf{0 . 0 6 0}$ \\
Reduced A/G ratio & 3.673 &
\end{tabular}

A/G: albumin to globulin ratio; CI: confidence interval. $\mathrm{P}$ values for significant variable $(P<0.2)$ are highlighted in bold font.

Table 5

Results of univariable Cox regression analysis of potential factors associated with survival at the $20 \%$ level $(P<0.2)$ at admission of 40 FeLV-seropositive cats.

\begin{tabular}{|c|c|c|c|c|}
\hline \multirow[t]{2}{*}{ Variable } & \multicolumn{4}{|c|}{ 95.0\% CI for Hazard Ratio } \\
\hline & $\begin{array}{l}\text { Hazard } \\
\text { Ratio }\end{array}$ & Lower & Upper & $\begin{array}{l}P \\
\text { value }\end{array}$ \\
\hline Male sex & 0.941 & 0.389 & 2.276 & 0.892 \\
\hline $\begin{array}{l}\text { Reduced RBCs } \\
\text { count }\end{array}$ & 3.355 & 1.263 & 8.913 & 0.015 \\
\hline Leucopenia & 1.048 & 0.416 & 2.636 & 0.921 \\
\hline Neutropenia & 1.103 & 0.408 & 2.981 & 0.846 \\
\hline Lymphopenia & 1.773 & 0.396 & 7.950 & 0.454 \\
\hline Hypercreatininemia & 0.855 & 0.188 & 3.888 & 0.840 \\
\hline Reduced $\mathrm{A} / \mathrm{G}$ ratio & 0.818 & 0.230 & 2.917 & 0.717 \\
\hline
\end{tabular}

A/G: albumin to globulin ratio; CI: confidence interval. P values for significant variable $(P<0.2)$ are highlighted in bold font.

hemolytic anemia, and is the cause of death in infected cats (Dean et al., 1992). In FeLV-seropositive cats with reduced RBC counts at diagnosis the death ratio was 3.5 times higher than in FeLV-seropositive cats with normal RBC counts at diagnosis. Presence of anemia was therefore a negative prognostic indicator for survival in FeLV-seropositive cats. These results are in agreement with a recent study in which cats that developed non-regenerative anemia following persistent infection after experimental FeLV infection lived an average of only 0.6 years (from 0.6 to 1.1 years) whereas cats that developed lymphoma survived on average 3.6 years (from 1.6 to 6.5 years) (Helfer-Hungerbuehler et al., 2015).

We identified a number of limitations of this study. Firstly, as with all retrospective studies, some cases had missing data. Laboratory data were not available for 14 retrovirus-infected cats and therefore these seropositive cats were not included in the study population. The number of cats in seropositive group cats was low. With a small sample size population, differences can be hard to detect statistically. In addition, relatively high numbers of retrovirus-seropositive cats were censored from the survival analysis because they were still alive or were lost to follow up at the time of completion of the study. This may have biased the results and should be noted when interpreting the data. Despite these drawbacks, studies of natural infection provide information relevant for practitioners faced with retrovirus-infected cats.

In our study, no true confirmation tests such as Western blot for FIV and immunofluorescent antibody (IFA), polymerase chain reaction (PCR) or virus isolation for FeLV were performed. Because positive assay results were not confirmed by means of an alternative assay, it is possible that some positive assay results were false positive. However, a recently study reported that the ELISA test kit used in our investigation showed excellent performance in diagnosis of these retroviruses (Levy et al., 2017).

Through the study we use the term seropositive for the study population. In FeLV infection, persistent antigenemia is consistent with progressive infection. In addition, since whole blood is currently the recommended specimen for patient-side FeLV testing, PCR detection of DNA provirus remains the gold standard confirmatory test to definitively diagnose FeLV infection and PCR testing should be pursued to confirm any positive p27 antigen test result (Westman et al., 2017). As cats in this study were not tested twice nor tested with PCR, we cannot be sure that they were all retrovirus infected cats.

Regardless of the performances of the test used, false positive results may occur especially in very young animals when maternal anti-FIV antibodies are still present or due to cross-reactions. In animals $<6$ months old there is a risk that maternal antibodies could interfere with the FIV antibody ELISA to give false positive results. However there were few very young cats in our study so this factor is unlikely to have biased our results. In addition there is no commercially available FIV vaccine in Europe so overestimation of FIV seroprevalence due to vaccine-induced FIV antibodies was not possible (Levy et al., 2004).

Furthermore, false negatives may also occur as FIV-infected cats do not develop antibodies for 2-4 weeks (or up to a year in some cases)

Table 6

Results of univariable Cox regression analysis of potential factors associated with survival at the $20 \%$ level $(P<0.2)$ at admission of 10 FIV + FeLV-seropositive cats.

\begin{tabular}{|c|c|c|c|c|}
\hline \multirow[t]{2}{*}{ Variable } & \multicolumn{4}{|c|}{ 95.0\% CI for Hazard Ratio } \\
\hline & Hazard Ratio & Lower & Upper & $P$ value \\
\hline Male sex & 1.261 & 0.247 & 6.451 & 0.780 \\
\hline Reduced RBCs count & 106.922 & 0.026 & 437806.644 & 0.271 \\
\hline Leucopenia & 0.299 & 0.034 & 2.604 & 0.274 \\
\hline Neutropenia & - & - & - & - \\
\hline Lymphopenia & - & - & - & - \\
\hline Hypercreatininemia & 1.274 & 0.208 & 7.797 & 0.793 \\
\hline Reduced $\mathrm{A} / \mathrm{G}$ ratio & 1.103 & 0.151 & 8.082 & 0.923 \\
\hline
\end{tabular}

- These categories were excluded from analyses because of small numbers.

Table 7

The final logistic regression model for factors associated with survival time in 53 FIV-seropositive and 40 FeLV-seropositive cats.

\begin{tabular}{|c|c|c|c|c|c|c|c|}
\hline Variable & Infection & B & Standard error & Wald & $P$ value & Hazard Ratio & 95.0\% CI for Hazard Ratio \\
\hline \multirow[t]{2}{*}{ Reduced RBCs count } & FIV & 2.4 & 0.7 & 9.3 & 0.002 & 11.0 & $2.3-51.8$ \\
\hline & FeLV & 1.2 & 0.5 & 5.5 & 0.015 & 3.5 & $1.2-10.0$ \\
\hline
\end{tabular}




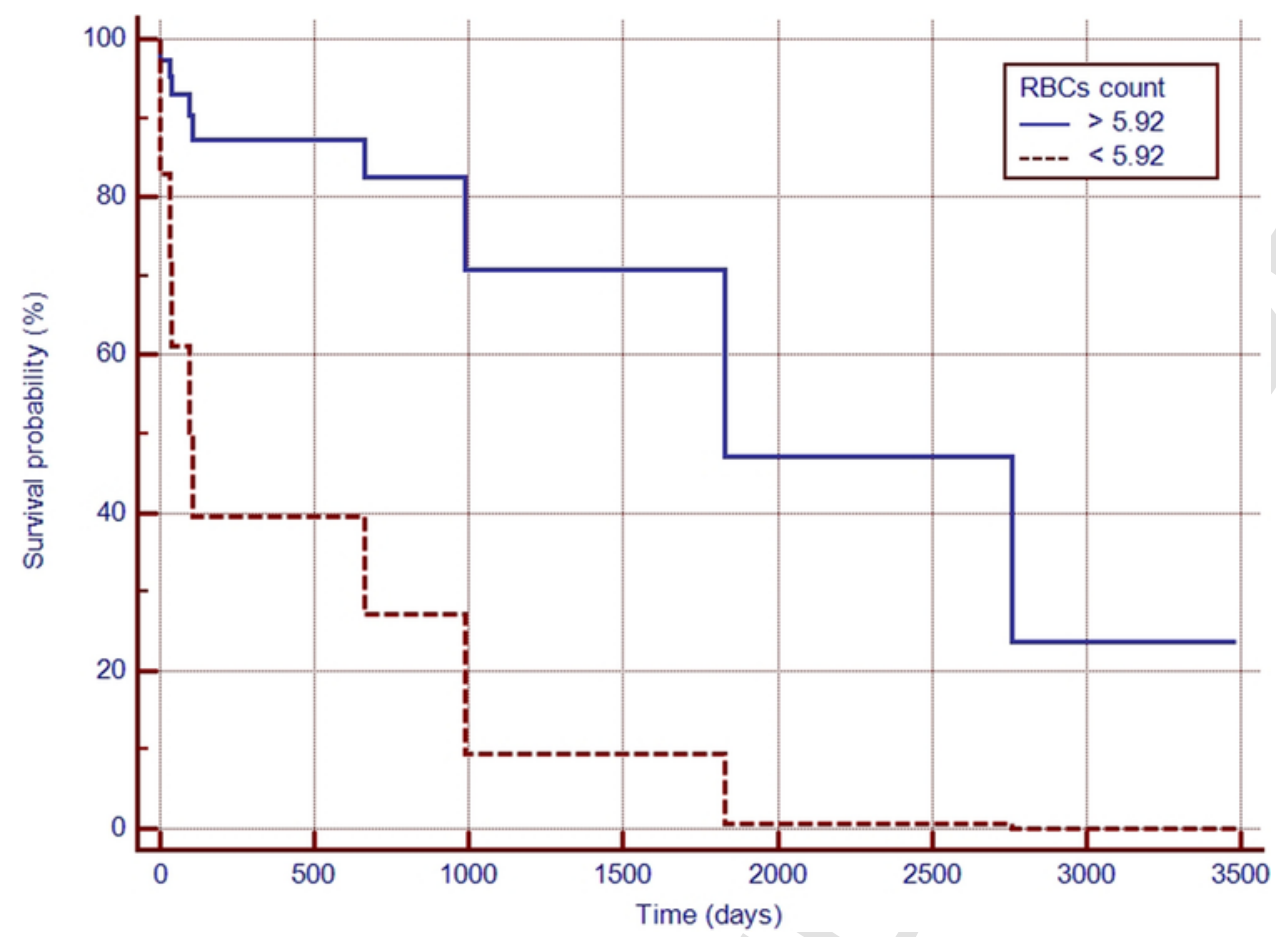

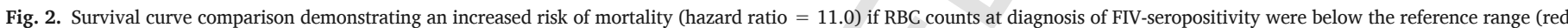
broken line). (For interpretation of the references to colour in this figure legend, the reader is referred to the web version of this article.)

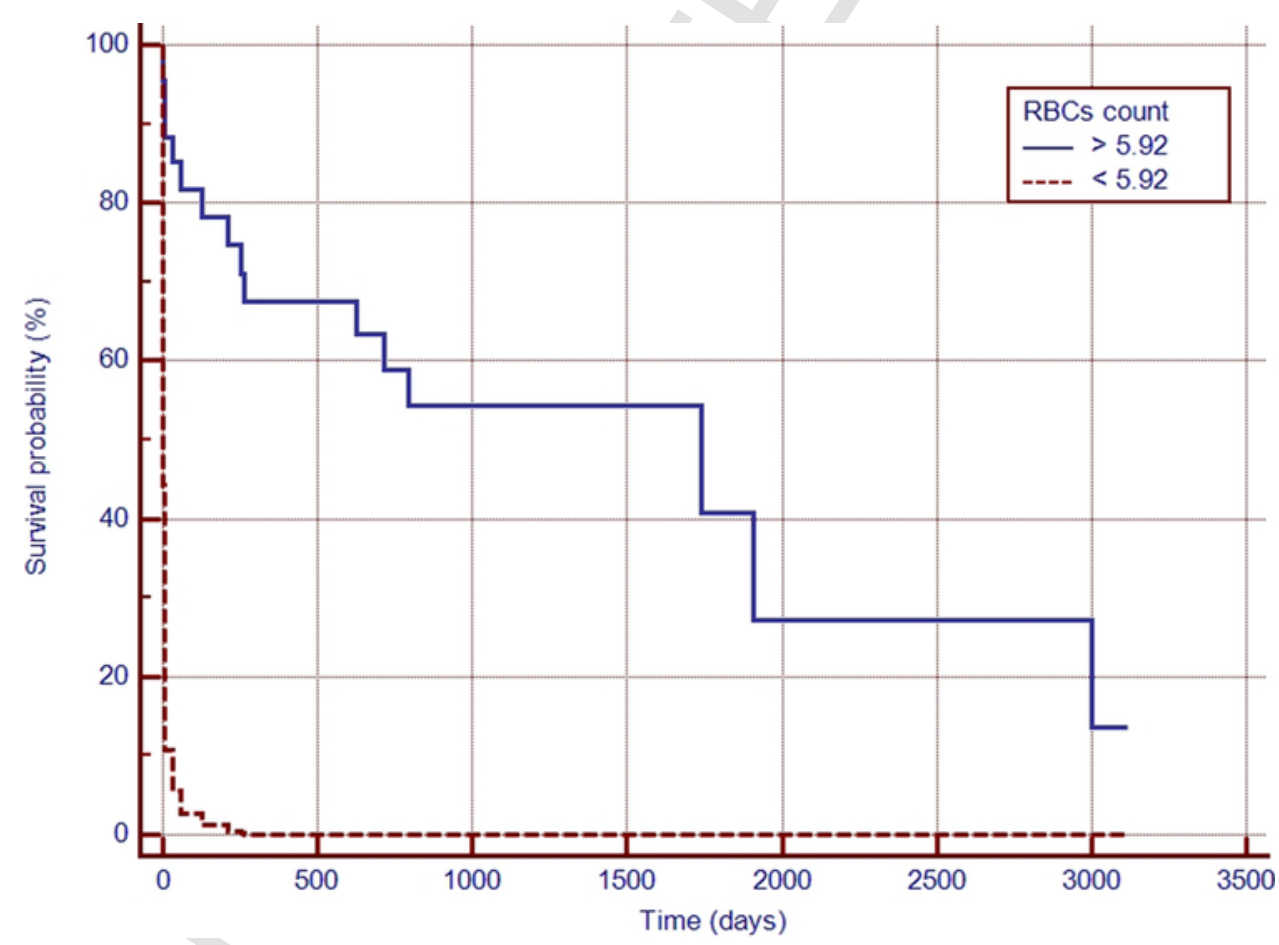

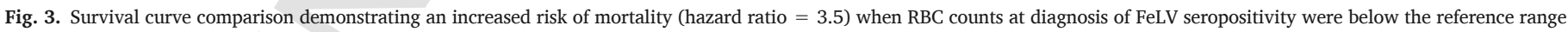
(red broken line). (For interpretation of the references to colour in this figure legend, the reader is referred to the web version of this article.)

post-infection or during the terminal stages of infection or in leukopenia (Swango, 1991).

Similarly, FeLV antigen may not be detected in latent FeLV infections when there is no viremia but the viral genome is integrated in bone marrow or other sites (Herring et al., 2001). However, the results of this study are based on field-based data generated from routine prac- tices and the use of these data is justified by the production of results that reflect the situation in the clinical field

\section{Conclusions}

In summary, FIV status did not significantly affect longevity of cats in this study, but FeLV and FIV-FeLV co-infection did. FIV and FeLV- 
seropositive cats with low RBC counts at diagnosis had significantly shorter survival times. It is therefore important to assess blood counts in retrovirus infected cats to provide specific information on prognosis. New treatment strategies are necessary for cats with retrovirus-associated anemia.

\section{Conflicts of interest}

None.

\section{Funding}

This research did not receive any specific grant from funding agencies in the public, commercial or not-for-profit sectors.

\section{References}

Ackley, C.D., Yamamoto, J.K., Levy, N., Pedersen, N.C., Cooper, M.D., 1990. Immunologic abnormalities in pathogen-free cats experimentally infected with feline immunodeficiency virus. J. Virol. 64, 5652-5655.

Addie, D.D., Toth, S., Reid, S., Jarrett, O., Dennis, J.M., Callanan, J.J., 2000. Long-term impact on a closed household of pet cats of natural infection with feline coronavirus, feline leukaemia virus and feline immunodeficiency virus. Vet. Rec. 146, 419-424. https://doi.org/10.1136/vr.146.15.419.

Arjona, A., Escolar, E., Soto, I., Barquero, N., Martin, D., Gomez-lucia, E., 2000. Seroepidemiological survey of infection by feline leukemia virus and immunodeficiency virus in Madrid and correlation with some clinical aspects. J. Clin. Microbiol. 38, 3448-3450.

Bachmann, M.H., Mathiason-Dubard, C., Learn, G.H., Rodrigo, A.G., Sodora, D.L., Mazzetti, P., Hoover, E.A., Mullins, J.I., 1997. Genetic diversity of feline immunodeficiency virus: dual infection, recombination, and distinct evolutionary rates among envelope sequence clades. J. Virol. 71, 4241-4253.

Baxter, K.J., Levy, J.K., Edinboro, C.H., Vaden, S.L., Tompkins, M.B., 2012. Renal disease in cats infected with feline immunodeficiency virus. J. Vet. Intern. Med. 26, 238-243. https://doi.org/10.1111/j.1939-1676.2011.00871.x.

Beczkowski, P.M., Litster, A., Lin, T.L., Mellor, D.J., Willett, B.J., Hosie, M.J., 2015. Contrasting clinical outcomes in two cohorts of cats naturally infected with feline immun odeficiency virus (FIV). Vet. Microbiol. 176, 50-60. https://doi.org/10.1016/j.vetmic. 2014.12.023.

Burling, A.N., Levy, J.K., Scott, H.M., Crandall, M.M., Tucker, S.J., Wood, E.G., Foster, J.D., 2017. Seroprevalences of feline leukemia virus and feline immunodeficiency virus infection in cats in the United States and Canada and risk factors for seropositivity. J. Am. Vet. Med. Assoc. 251, 187-194.

Chhetri, B.K., Berke, O., Pearl, D.L., Bienzle, D., 2015. Comparison of risk factors for seropositivity to feline immunodeficiency virus and feline leukemia virus among cats: a case-case study. BMC Vet. Res. 11, 30. https://doi.org/10.1186/ s12917-015-0339-3.

Dean, G.A., Groshek, P.M., Mullins, J.I., Hoover, E.A., 1992. Hematopoietic target cells of anemogenic subgroup $\mathrm{C}$ versus nonanemogenic subgroup A feline leukemia virus. J. Virol. 66, 5561-5568.

Flynn, J.N., Cannon, C.A., Lawrence, C.E., Jarrett, O., 1994. Polyclonal B-cell activation in cats infected with feline immunodeficiency virus. Immunology 81, 626-630.

Fujino, Y., Horiuchi, H., Mizukoshi, F., Baba, K., Goto-Koshino, Y., Ohno, K., Tsujimoto, H., 2009. Prevalence of hematological abnormalities and detection of infected bone marrow cells in asymptomatic cats with feline immunodeficiency virus infection. Vet. Microbiol. 136, 217-225. https://doi.org/10.1016/j.vetmic.2008.11.007.

Garigliany, M., Jolly, S., Dive, M., Bayrou, C., Berthemin, S., Robin, P., Godenir, R., Petry, J., Dahout, S., Cassart, D., Thiry, E., Desmecht, D., Saegerman, C., 2016. Risk factors and effect of selective removal on retroviral infections prevalence in Belgian stray cats. Vet. Rec. 178, 45. https://doi.org/10.1136/vr.103314.

Gates, M.C., Vigeant, S., Dale, A., 2017. Prevalence and risk factors for cats testing positive for feline immunodeficiency virus and feline leukaemia virus infection in cats entering an animal shelter in New Zealand. N. Z. Vet. J. https://doi.org/10.1080/00480169. 2017.1348266.

Giordano, A., Paltrinieri, S., 2010. Interpretation of capillary zone electrophoresis compared with cellulose acetate and agarose gel electrophoresis: reference intervals and diagnostic efficiency in dogs and cats. Vet. Clin. Pathol. 39, 464-473. https://doi.org/ 10.1111/j.1939-165X.2010.00255.x.

Gleich, S., Hartmann, K., 2009. Hematology and serum biochemistry of feline immunodeficiency virus-infected and feline leukemia virus-infected cats. J. Vet. Intern. Med. 23, 552-558. https://doi.org/10.1111/j.1939-1676.2009.0303.x.

Gleich, S.E., Krieger, S., Hartmann, K., 2009. Prevalence of feline immunodeficiency virus and feline leukaemia virus among client-owned cats and risk factors for infection in Germany. J. Feline Med. Surg.https://doi.org/10.1016/j.jfms.2009.05.019.

Gleich, S.E., Krieger, S., Hartmann, K., 2009. Prevalence of feline immunodeficiency virus and feline leukaemia virus among client-owned cats and risk factors for infection in Germany. J. Feline Med. Surg. 11, 985-992. https://doi.org/10.1016/j.jfms.2009.05. 019.

Helfer-Hungerbuehler, A.K., Widmer, S., Kessler, Y., Riond, B., Boretti, F.S., Grest, P., Lutz, H., Hofmann-Lehmann, R., 2015. Long-term follow up of feline leukemia virus infection and characterization of viral RNA loads using molecular methods in tissues of cats with different infection outcomes. Virus Res. 197, 137-150. https://doi.org/10.1016/ j.virusres.2014.12.025.
Herring, E.S., Troy, G.G., Toth, T.E., Forrester, S.D., Weigt, L.A., Herring, I.P., 2001. Detection of feline leukaemia virus in blood and bone marrow of cats with varying suspicion of latent infection. J. Feline Med. Surg. 3, 133-141. https://doi.org/10.1053/ jfms.2001.0132.

Hofmann-Lehmann, R., Holznagel, E., Aubert, A., Ossent, P., Reinacher, M., Lutz, H., 1995 Recombinant FeLV vaccine: long-term protection and effect on course and outcome of FIV infection. Vet. Immunol. Immunopathol. 46, 127-137. https://doi.org/10.1016/ 0165-2427(94)07012-V.

Hofmann-Lehmann, R., Holznagel, E., Ossent, P., Lutz, H., 1997. Parameters of disease progression in long-term experimental feline retrovirus (feline immunodeficiency virus and feline leukemia virus) infections: hematology, clinical chemistry, and lymphocyte subsets. Clin. Diagn. Lab. Immunol. 4, 33-42. https://doi.org/10.1016/j.jfms. 2009.05.006.

Klein, J., Moeschberger, M., 2003. Survival Analysis. Techniques for Censored and Truncated Data, 2nd ed. Springer Publishers, New York.

Kohmoto, M., Uetsuka, K., Ikeda, Y., Inoshima, Y., Shimojima, M., Sato, E., Inada, G., Toyosaki, T., Miyazawa, T., Doi, K., Mikami, T., 1998. Eight-year observation and comparative study of specific pathogen-free cats experimentally infected with feline immunodeficiency virus (FIV) subtypes A and B: terminal acquired immunodeficiency syndrome in a cat infected with FIV petaluma strain. J. Vet. Med. Sci. 60, 315-321. https://doi.org/10.1292/jvms.60.315.

Levy, J.K., Crawford, P.C., Slater, M.R., 2004. Effect of vaccination against feline immun odeficiency virus on results of serologic testing in cats. J. Am. Vet. Med. Assoc. 225, 1558-1561. https://doi.org/10.2460/javma.2004.225.1558.

Levy, J.K., Scott, H.M., Lachtara, J.L., Crawford, P.C., 2006. Seroprevalence of feline leukemia virus and feline immunodeficiency virus infection among cats in North America and risk factors for seropositivity. J. Am. Vet. Med. Assoc. 228, 371-376. https://doi.org/10.2460/javma.228.3.371.

Levy, J., Crawford, C., Hartmann, K., Hofmann-Lehmann, R., Little, S., Sundahl, E., Thayer, V., 2008. 2008 American Association of Feline Practitioners' feline retrovirus management guidelines. J. Feline Med. Surg. 10, 300-316. https://doi.org/10.1016/ j.jfms.2008.03.002.

Levy, J.K., Crawford, P.C., Tucker, S.J., 2017. Performance of 4 point-of-care screening tests for feline leukemia virus and feline immunodeficiency virus. J. Vet. Intern. Med. 31, 521-526. https://doi.org/10.1111/jvim.14648.

Liem, B.P., Dhand, N.K., Pepper, A.E., Barrs, V.R., Beatty, J.A., 2013. Clinical findings and survival in cats naturally infected with feline immunodeficiency virus. J. Vet. Intern. Med. 27, 798-805. https://doi.org/10.1111/jvim.12120.

Lutz, H., Addie, D., Belak, S., 2009. Feline Leukemia ABCD guidelines on prevention and management. J. Feline Med. Surg. 11, 565-574.

Mirò, G., Doménech, A., Escolar, E., Collado, V.M., Tejerizo, G., De Las Heras, A., Gòmez-Lucìa, E., 2007. Plasma electrophoretogram in feline immunodeficiency virus (FIV) and/or feline leukaemia virus (FeLV) infections. J. Vet. Med. Ser. A: Physiol. Pathol. Clin. Med. 54, 203-209. https://doi.org/10.1111/j.1439-0442.2007.00902.x.

Moritz, A., Fickenscher, Y., Meyer, K., Failing, K., Weiss, D.J., 2004. Canine and feline hematology reference values for the ADVIA 120 hematology system. Vet. Clin. Pathol. 33, 32-38. https://doi.org/10.1111/j.1939-165X.2004.tb00347.x.

Murray, J.K., Roberts, M.A., Skillings, E., Morrow, L.D., Gruffydd-Jones, T.J., 2009. Risk factors for feline immunodeficiency virus antibody test status in Cats Protection adoption centres (2004). J. Feline Med. Surg. 11, 467-473. https://doi.org/10.1016/j.jfms. 2008.11.001.

Pedersen, N.C., Torten, M., Rideout, B., Sparger, E., Tonachini, T., Luciw, P.A., Ackley, C., Levy, N., Yamamoto, J., 1990. Feline leukemia virus infection as a potentiating cofactor for the primary and secondary stages of experimentally induced feline immunodeficiency virus infection. J. Virol. 64, 598-606.

Peri, E.V., Ponti, W., Dall'ara, P., Rocchi, M., Zecconi, A., Bonizzi, L., 1994. Seroepidemiological and clinical survey of feline immunodeficiency virus infection in northern Italy. Vet. Immunol. Immunopathol. 40, 285-297.

Pistello, M., Cammarota, G., Nicoletti, E., Matteucci, D., Curcio, M., Del Mauro, D., Bendinelli, M., 1997. Analysis of the genetic diversity and phylogenetic relationship of Italian isolates of feline immunodeficiency virus indicates a high prevalence and heterogeneity of subtype B. J. Gen. Virol. 78, 2247-2257. https://doi.org/10.1099/ 0022-1317-78-9-2247.

Poli, A., Tozon, N., Guidi, G., Pistello, M., 2012. Renal alterations in feline immunodeficiency virus (FIV)-infected cats: a natural model of lentivirus-induced renal disease changes. Viruses 4, 1372-1389. https://doi.org/10.3390/v4091372.

Ravi, M., Wobeser, G.A., Taylor, S.M., Jackson, M.L., 2010. Naturally acquired feline immunodeficiency virus (FIV) infection in cats from western Canada: prevalence, disease associations, and survival analysis. Can. Vet. J. 51, 271-276.

Shelton, G.H., Grant, C.K., Cotter, S.M., Gardner, M.B., Hardy, W.D., DiGiacomo, R.F., 1990. Feline immunodeficiency virus and feline leukemia virus infections and their relationships to lymphoid malignancies in cats: a retrospective study (1968-1988). J. Acquir. Immune Defic. Syndr. 3, 623-630.

Shelton, G.H., Linenberger, M.L., Persik, M.T., Abkowitz, J.L., 1995. Prospective hematologic and clinicopathologic study of immunodeficiency virus infection. J. Vet. Intern. Med. 9, 133-140.

Silverstein, D.C., Hopper, K., 2015. Small Animal Critical Care Medicine, 2nd ed. Elsevier Saunders, St Louis, Missouri.

Spada, E., Proverbio, D., Della Pepa, A., Perego, R., Baggiani, L., De Giorgi, G.B., Domenichini, G., Ferro, E., Cremonesi, F., 2012. Seroprevalence of feline immunodeficiency virus, feline leukaemia virus and Toxoplasma gondii in stray cat colonies in north ern Italy and correlation with clinical and laboratory data. J. Feline Med. Surg. 14, 369-377. https://doi.org/10.1177/1098612X12437352.

Sparkes, A.H., Hopper, C.D., Millard, W.G., Gruffydd-Jones, T.J., Harbour, D.A., 1993. Feline immunodeficiency virus infection. Clinicopathologic findings in 90 naturally occurring cases. J. Vet. Intern. Med. 7, 85-90.

Swango, L.J., 1991. Evaluation of feline leukemia virus diagnostic tests available for in-office use by veterinarians. J. Am. Vet. Med. Assoc. 199, 1386-1389. 
Sykes, J.E., Hartmann, K., 2014. Feline leukemia virus infection. In: Sykes, J.E. (Ed.), Canine and Feline Infectious Diseases.. Elsevier Saunders, St Louis Missouri, pp. 224-238.

Vail, D.M., Moore, A.S., Ogilvie, G.K., Volk, L.M., Samples, T., 1998. Feline lymphoma (145 Cases): proliferation indices, cluster of differentiation 3 immunoreactivity, and their association with prognosis in 90 cats. J. Vet. Intern. Med. 12, 349-354.

Westman, M.E., Paul, A., Malik, R., McDonagh, P., Ward, M.P., Hall, E., Norris, J.M., Westman, M.E., Norris, J.M., 2016. Seroprevalence of feline immunodeficiency virus and feline leukaemia virus in Australia: risk factors for infection and geographical influences (2011-2013). J. Feline Med. Surg. Open Rep. 2, 1-11. https://doi.org/10.1177/ 2055116916646388.

Westman, M.E., Malik, R., Hall, E., Sheehy, P.A., Norris, J.M., 2017. Comparison of three feline leukaemia virus (FeLV) point-of-care antigen test kits using blood and saliva. Comp. Immunol. Microbiol. Infect. Dis. 50, 88-96. https://doi.org/10.1016/j.cimid. 2016.11.014. 